\title{
The convergence and divergence of episodic and semantic functions across lateral parietal cortex
}

\author{
Gina F. HumPhreYs ${ }^{1}$, JeYoung Jung ${ }^{2}$, \& Matthew A. LAMBOn RALPH ${ }^{1}$
}

1. MRC Cognition \& Brain Sciences Unit, University of Cambridge, Cambridge, UK

2. School of Psychology, University of Nottingham, UK

Address for correspondence:

Dr. Gina Humphreys (Gina.Humphreys@mrc-cbu.cam.ac.uk) or Prof. Matt Lambon Ralph (Matt.Lambon-Ralph@mrc-cbu.cam.ac.uk)

MRC Cognition and Brain Sciences Unit

University of Cambridge

15 Chaucer Road

Cambridge

$\mathrm{CB} 27 \mathrm{EF}$

UK

Tel: +44 (0)1223355294

Keywords: Parietal, episodic, semantic, angular gyrus, fMRI 


\begin{abstract}
Several decades of neuropsychological and neuroimaging research have highlighted the importance of lateral parietal cortex (LPC) across a myriad of cognitive domains. Yet, despite the prominence of this region the underlying function of LPC remains unclear. Two domains that have placed particular emphasis on LPC involvement are semantic memory and episodic memory retrieval. From each domain, sophisticated models have been proposed as to the underlying function, as well as the more domain-general that LPC is engaged by any form of internally-directed cognition (episodic and semantic retrieval both being examples if this process). Here we directly address these alternatives using a combination of fMRI and DTI white-matter connectivity data. The results show that ventral LPC (angular gyrus) was positively engaged during episodic retrieval but disengaged during semantic memory retrieval. In addition, the level of activity negatively varied with task difficulty in the semantic task whereas episodic activation was independent of difficulty. In contrast, dorsal LPC (intraparietal sulcus) showed domain general activation that was positively correlated with task difficulty. In terms of structural connectivity, a dorsal-ventral and anterior-posterior gradient of connectivity was found to different processing networks (e.g., mid-angular gyrus (AG) connected with episodic retrieval). We propose a unifying model in which LPC as a whole might share a common underlying function (e.g., multimodal buffering) and variations across subregions arise due to differences in the underlying white matter connectivity.
\end{abstract}




\section{Introduction}

Several decades of neuropsychological and neuroimaging research have highlighted the importance of lateral parietal cortex (LPC) across a myriad of cognitive domains (1-6). It also forms a core part of the default mode network (DMN), a network that often deactivates during taskperformance (7-9) Yet, despite the prominence of this region in basic and clinical research, the underlying function of LPC remains unclear. Part of this confusion may reflect that cognitive neuroscience research is often focused and organised by the cognitive domain of interest. As a result, multiple cognitive domains have been associated with the LPC with minimal cross-talk between these separate literatures and resultant cognitive neuroscience theories (with a few notable exceptions: (1-3) Whilst these theories are often sophisticated and are based on a wealth of domain-specific data, they fail to explain both the wide variety of functions that appear to recruit this region and, thus, what the core underpinning neurocomputations might be. There are two types of answer to this fundamental question $(1,10)$ 1) a form of 'neuromarquetry' in which LPC contains numerous sub-regions each recruited by different tasks and serve distinct underlying cognitive functions; or 2) multiple cognitive activities rely, in common, upon a small number of underlying computations that arise from the LPC and its patterns of connectivity to the wider neural network (11-14). Fathoming the nature of LPC neurocomputations (and by extension other higher cortical regions) will require a different approach. Rather than focus solely upon an individual cognitive domain, it will be necessary to combine data across multiple higher cortical functions simultaneously. The current study adopted this approach by directly comparing two cognitive domains (semantic and episodic memory), as well as the more domain-general that LPC is engaged by any form of internally-directed cognition (episodic and semantic retrieval both being examples if this process). Despite being associated with the LPC (and, in particular, the angular gyrus) throughout long neuropsychological and functional neuroimaging literatures, they have rarely been directly compared in the same group of participants (and those that have been conducted have suffered from some important limitations). The current study not only met this novel aim but also subsequently explored the identified key LPC subregions through analyses of DTI white-matter connectivity data.

\section{Domain-specific theories}

Many LPC theories and datasets focussed on individual cognitive domains have been presented. The semantic hypothesis has a very long history and tradition in both neuropsychology and from the start of functional neuroimaging. According to this proposal, the ventral LPC, specifically the angular gyrus (AG), acts as a semantic-hub which stores multi-modal semantic information $(5,15)$ The observations of concrete $>$ abstract and words $>$ nonword differences in the AG found in individual studies and in formal meta-analyses form a cornerstone of evidence for this proposal. In contrast, numerous studies have also observed AG responses during episodic retrieval, with activation typically correlating the vividness of the memory retrieved. This has led to the suggestion that LPC might act 
as some form of episodic buffer $(6,16)$. The fact that these two very different components of human long-term memory seem to involve the same brain LPC region has received little attention despite the very large and growing number of studies on each one. In fact, the situation for episodic and semantic memory is a worked example of the broader challenge - namely that many different cognitive domains have been implicated and do overlap in the LPC, yet few explanations have been offered for this conundrum $(1,10,17)$.

\section{Domain-general theories}

A handful of research groups have noted this confluence of multiple cognitive functions in the LPC. The resultant theories argue that some LPC processes might be domain-general in nature and represent more fundamental neurocomputations that are required by multiple cognitive activities (1-3, 18-20). For instance, there is good evidence to suggest that a common region within dorsal LPC (intraparietal sulcus (IPS)) forms part of a "multiple demand network" and is recruited as part of a fronto-parietal network for executive processing $(1,18,20)$ Going further, several theories suggest that dorsal LPC (dLPC) within the IPS, and ventral LPC (vLPC) serve counterpointed functions that are utilised across cognitive domains. The dLPC has been implicated in any task that requires topdown attentional control whereas ventral areas are automatically recruited for bottom-up attention (2) Relatedly, dLPC is involved in tasks that require externally-directed attention, whereas vLPC is active during internally-directed attention, as is the case when retrieving semantic and/or episodic memories, as well as other processes such as future planning or self-projection $(7,21)$. Since these internallydirected processes are not required during most fMRI tasks the vLPC is deactivated, hence its involvement in the DMN.

\section{Graded-variability and PUCC}

In terms of underlying architecture, anatomical evidence suggests that, rather than sharply defined tessellations across the LPC, anatomical variations appear to be graded in nature (11-14). Consequently, at least some graded variability in cognitive function across LPC might be expected. The PUCC model suggests that, whilst LPC as a whole might share a common underlying function (e.g., multimodal buffering), variations across subregions will arise due to differences in the underlying white matter connectivity (1). Going beyond previous large-scale meta-analyses and reviews $(1,10,17)$ the current study served two purposes: 1$)$ to compare alternative theories of LPC function directly within the same group of participants (which is not possible in large-scale metaanalyses), and 2) to examine the extent to which variations in activation patterns could arise from underlying structural variations white-matter connectivity. 
Previous studies have demonstrated that, in order to explore LPC functions and reveal interpretable findings, it is necessary to take certain factors into account within the design and analysis of any study:

1) The direction of activation relative to rest: given the involvement of LPC in the $\mathrm{DMN}$, it is of critical importance to consider whether a task positively or negatively engages the LPC relative to rest. Whilst many tasks generate deactivation in the AG, this is not always the case and the handful of activities that do positively engage the AG might be crucial sources of evidence about its true contribution (1). Contrasts between a cognitive task of interest vs. an active control condition are ambiguous because the difference could result from 1) greater positive activation for the task or 2) greater deactivation for the control. This issue becomes even more important when considering the impact of task difficulty on activation and deactivation in this region (see next). A straightforward expectation applied to almost all brain regions is that if a task critically requires the LPC then the LPC should be positively engaged by that task relative to baseline.

2) Task-difficulty: Task-difficulty is important in two different ways. First, task difficulty correlates positively with activation in dLPC (dorsal AG/IPS) but negatively with the level of activation within vLPC or, put in a different way, the level of deactivation in vLPC (mid-AG) is positively related to task difficulty. Indeed, the dLPC and VLPC have often been shown to be anticorrelated in resting state data $(18,22-24)$. Secondly, task-difficulty deactivations need to be accounted for when interpreting differences in ventral LPC areas. A 'positive' difference can be obtained in the AG simply by comparing easy $>$ hard task conditions even for tasks that are entirely non-semantic, non-linguistic and non-episodic (18). One major limitation of evidence in favour of the semantic hypothesis is that apparent semantic effects observed from fMRI studies could be explained by a difficulty confound (e.g. word $>$ nonword, concrete $>$ abstract). Indeed, it has been shown that one can both eliminate the difference between semantic and non-semantic tasks when task difficulty is controlled (18) and, more compellingly, also flip the typical 'semantic' effects (e.g. words > nonwords, concrete $>$ abstract) by reversing the difficulty of the tasks or stimuli $(25,26)$.

3) The importance of within-study comparisons: Reviews and formal meta-analyses of existing fMRI data have clearly identified overlapping areas of activations within the LPC (1). Whilst highly suggestive of domain-general computations in these regions, one needs within-participant comparisons to test these hypotheses further. Without such evidence, two alternative interpretations are possible: 1) True overlap across tasks implicating the region in a common neurocomputation (1-3, $18-20$ ) or 2) small-scale variability in function across the LPC which is blurred by cross-study comparisons or meta-analyses (27)

The current study sought to determine a unifying theory of LPC function using a combination of fMRI and DTI white-matter connectivity data. We tested the hypotheses that the LPC shares a common underlying function but has graded subregions arising from variations in the underlying 
connectivity profiles. To test this, we contrasted several key fMRI tasks in a direct within-study comparison, as well as determining the underlying white matter connectivity profile of the identified LPC subregions. Following some of the dominant proposals about LPC function (reviewed above), we manipulated internally vs. externally directed attention, and episodic retrieval vs. semantic retrieval. Importantly, we considered the direction of activation/deactivation vs. rest as well as the extent to which the results can be explained in terms of variations in task-difficulty. There were four conditions, two involving internally-directed attention (semantic or episodic retrieval) and two involving externally-directed visual attention (real-world object decision or scrambled pattern decision). In each task the participant was presented with word-triads including a target word in the centre of the screen and two words below. In the semantic task, the participants indicated which item was semantically related to the target (e.g. daffodil: yellow or red). In the episodic task, the participants selected the feature that best matched the target items. The target items were verbal labels that corresponded to colour photographs that were viewed prior to the scan (e.g. bucket: green or red). Vividness ratings were made after each trial. In the object-decision tasks participants responded to question about a picture presented on the screen (chair: blue red), and the control task they indicated the direction of a scrambled picture that was shifted to the left or right side of the screen (see Figure 1). We also used DTI to examine variations in the underlying structural connectivity profiles from LPC subregions to rest of the brain.

\section{Results}

\section{Behavioural results}

On average the participants produced relatively few incorrect responses (average proportion incorrect (SD): episodic task $=0.13(.05)$, semantic task $=0.06(0.4)$, picture task $=0.09(.03)$, and control task $=0.01(0.1))$ (see Figure 3). A one-way within-subjects ANOVA revealed that the tasks varied significantly in difficulty $(\mathrm{F}(21)=111.88, \mathrm{p}<.001)$. Pairwise comparisons showed that the episodic task was significantly more difficult than all other tasks (all $t \mathrm{~s}>3$, all $p \mathrm{~s}<.006$ ), the picture task was harder than the semantic task and control task (all $t \mathrm{~s}>3$, all $p \mathrm{~s}<.005$ ), and the control task was easier than all other tasks ( $t \mathrm{~s}>3$, all $p \mathrm{~s}<.001$ ). Given the known effects of task difficulty on IPS activation and AG deactivation, then - all other things being equal - one might have expected the greatest IPS activation, and the greatest AG deactivation for the episodic task followed by the picture task, then semantic and final the control task. Of theoretical relevance, whilst this was true in the IPS, this pattern was not the observed in AG.

\section{fMRI results}

Whole-brain analyses were performed using a standard voxel height threshold $\mathrm{p}<.001$, cluster corrected using FWE $\mathrm{p}<.05$. The episodic task $>$ control task was found to activate bilateral 
lateral and medial frontal areas, dorsal (IPS) and ventral LPC (AG), medial parietal (precuneus), and posterior and medial occipito-temporal areas (see Figure 2). As with many other tasks with written word stimuli $(28,29)$ the semantic task > control task activation was mainly left-sided but overlapped with the episodic task in the lateral and medial frontal cortex, and posterior occipito-temporal areas although the activation for the semantic task spread more anteriorly along fusiform gyrus into the anterior temporal lobe, in congruence with its established role in semantic processing (30). In contrast to the episodic task, no parietal activation was found for the semantic task (in fact the vLPC was more strongly activated by the control task relative to semantics) (Figure 2). Indeed, direct task comparisons showed these network differences to be statistically significant (Figure 2). Importantly, given the importance of the direction of activation relative to rest, the same AG region that is positively activated in the episodic task (episodic task > rest), is deactivated in the semantic task (rest $>$ semantic task) (Figure 2).

ROI analyses directly explored the pattern of activation within LPC. Within AG, the results provided strong support that this region is primarily involved in episodic retrieval, rather than any other task. Despite being the hardest task, AG was found to be significantly positively activated relative to rest during episodic processing, as demonstrated by a one-sample t-test $(\mathrm{t}(21)=2.30, \mathrm{p}=$. $03)$. In contrast, $A G$ showed significant deactivation relative to rest during semantic retrieval $(\mathrm{t}(21)=$ $-4.39, \mathrm{p}<.00)$ thus strongly contradicting the semantic hypothesis. Indeed, semantic activation in the AG was found to also be lower than even the control task which required no semantic processing $(\mathrm{t}(21)=-2.84, \mathrm{p}=.01)$ (Figure 3). This provides clear evidence that the AG is engaged by episodic retrieval, but is disengaged during semantic retrieval. In further support of the role of AG in episodic retrieval, the network was found to be highly correlated with the vividness ratings. A whole-brain analysis showed a strong correlation with vividness within lateral and medial parietal cortex, as well as medial frontal areas (Figure 2). This network shows close correspondence to the DMN.

The IPS was found to be positively activated relative to rest for all tasks (all ts $>5.44, \mathrm{p}<$ .000) (Figure 3). Indeed, activation closely mirrored task difficulty. To test this, we examined the positive and negative correlation with trial difficulty in a whole-brain analyses. This revealed a large network of dorsal parietal cortex, as well as lateral frontal, and posterior temporal areas were strongly positively correlated with task difficulty. In contrast, ventral parietal cortex, as well as the wider DMN in medial frontal and parietal cortex were strongly negatively related to task difficulty thus supporting the notion that this network is disengaged when a task becomes harder to perform (Figure 2). Note that the exception to this rule is during episodic retrieval, which was the hardest task but nevertheless most strongly engaged ventral parietal cortex. Indeed, when the same whole brain correlation analysis was performed but only including the episodic retrieval trials no correlation was found with task difficulty within the DMN (even at the very lenient threshold $p<.05$ uncorrected). 
To summarise, the AG was actively engaged by the episodic retrieval task, and this positively correlated with the vividness of the memory. In contrast, the semantic task, picture-decision task, and control task all resulted in AG deactivation, and the level of deactivation was proportionate to task difficulty. The IPS, showed the reverse response, with increasing activation for increased task difficulty.

\section{DTI results}

Dorsal connectivity: Consistent with the role of dLPC in executive processing the IPS ROI showed long-range connectivity with lateral frontal lobe, specifically, dorsolateral frontal cortex (DLPFC) and IFG (BA44) which are known to be involved in top-down executive control (Figure 4 , Table 1).

Anterior-posterior gradient within the ventral parietal lobes: Within the vLPC, there was a graded variation in connectivity between anterior and posterior parietal cortex control (Figure 4, Table 1). The anterior VLPC showed connectivity with the temporal lobe areas implicated in language processing $(5,30,31)$. In contrast, moving in a posterior direction, in mid vLPC the connectivity tended to become stronger with areas associated with the DMN and episodic memory, including the inferior temporal cortex and the hippocampus, as well as large portions of the precuneus $(32,33)$. Finally, the posterior vLPC connected with areas including the medial parietal cortex and occipital lobe associated with visual processing and spatial attention $(2,34)$.

\section{$\underline{\text { Discussion }}$}

Summary of main results: The dorsal and ventral LPC were positively engaged during episodic retrieval (but not in any other task), and this activation was found to correlate positively with the vividness of the episodic memory. The vLPC (AG) showed strong deactivation compared to rest during semantic memory retrieval (indeed the LPC was less engaged by the semantic task than the control task that involved little/no semantic processing). This provides compelling evidence against theories that posit a role in semantic processing or in all forms of internally-directed thought. In terms of task difficulty, with the exception of the episodic task, activation within the vLPC (AG), as part of the wider DMN, showed a strong negative correlation with reaction time thereby suggesting that this region is "turned-off" when a task becomes increasingly difficult. This is in contrast to the dLPC, as part of a wider multi-demand fronto-parietal network, which increased activation in relation to taskdifficulty. Critically, the episodic retrieval task was the only task to activate both the dLPC and vLPC regions concurrently. In terms of structural connectivity, dLPC forms part of a fronto-parietal network, whereas the vLPC showed an anterior-posterior variation in connectivity. Specifically, the mid vLPC region (corresponding to the mid-PGp subregion of AG associated with the DMN) connected with areas associated with episodic memory retrieval (hippocampus and precuneus). 
Together with the fMRI results, this suggests the mid vLPC (PGp) forms part of the episodic retrieval network $(32,33)$. In contrast the anterior vLPC $(\mathrm{PGa})$ showed structural connectivity with temporal lobe language processing areas $(5,30,31)$. Indeed, the same region has been shown to be positively engaged by tasks that involve sentence-level processing $(1,35,36)$. Whereas, the posterior vLPC (posterior PGp) connected with the occipital lobe and medial parietal areas associated with visual attention $(2,34)$, and this same region has been shown to be positively engaged by a picture-sequence task (35). Together these results fit with the PUCC model that suggests a graded change in the functional engagement of VLPC based on graded changes in the underlying structurally connectivity of the network (see Figure 4 for a schematic model).

\section{Implications for semantic theories of LPC function}

The current data provide clear evidence that the AG is not engaged during semantic retrieval. How can this result be aligned with evidence showing AG apparent sensitivity to semantic contrasts (5)? One key explanation is based on the type of contrast being performed: typical contrasts being words $>$ nonword, or concrete $>$ abstract. A meta-analysis of semantic vs. non-semantic studies that used other forms of contrast found no evidence of AG engagement despite engagement of the wider fronto-temporal semantic network (37), and another study found stronger AG engagement during nonverbal tongue movements compared to meaningful-speech (38). As highlighted in the Introduction, when interpreting these data it is important to take into account two variables: 1) the polarity of activation relative to rest and 2) difficulty-related differences.

With regard the first point, the semantic retrieval task showed deactivation relative to rest. It is of course difficult to interpret "rest", as it would likely involve spontaneous semantic and linguistic processing (39) (although one could also make the argument that the same is also true for episodic retrieval $(7,21)$ yet episodic memory tasks positively engage the $A G$, as demonstrated here and elsewhere (1)). Importantly, whilst AG deactivates for semantic and non-semantic tasks, other key semantic areas do not show the same pattern as AG; for instance, the anterior temporal lobe is positively engaged during semantic tasks but deactivated by non-semantic tasks $(8,18)$.

With regard the second point, AG deactivation is known to relate to task difficulty $(18,40$, 41), with increasing deactivation for harder tasks. Indeed, the contrast of word $>$ nonword and concrete $>$ abstract typically involve comparing an easier task vs. a harder task. Compellingly, the same word $>$ nonword and concrete $>$ abstract contrasts can be inverted by reversing the difficulty of the task/stimuli $(25,26)$. Indeed, when task difficulty is directly manipulated in a semantic and visuospatial task one observes a main effect of task difficulty (easy vs. hard) in the AG but no semantic vs. non-semantic difference, whereas the IPS shows the reverse pattern of difficulty-related activation (hard vs. easy)(18).

\section{Implications for episodic memory retrieval:}


The episodic retrieval task showed positive activation in both the AG and IPS despite being the most difficult task; making it the exception to the difficulty-related activation pattern. The influence of connectivity into various IPL areas, embraced in the PUCC framework (see below), can explain these episodic findings in the ventral and dorsal parietal cortex. Specifically, the mid-PGp region of the AG connects with the hippocampus and precuneus, operating as part of a wider episodic retrieval network. Based on evidence that the patients with AG damage are not profoundly amnesic, unlike those with damage to the medial temporal lobe $(10,42,43)$, we propose that episodic information stored elsewhere in the system is temporally buffered online during episodic retrieval. Indeed the notion of an AG episodic buffer has been proposed elsewhere $(6,16)$. This notion is consistent with observation that patients with parietal damage lack clarity or vividness of episodic memories, as one might predict from a deficit in buffering multi-modal contextual information (4449). In addition, due to its connectivity with DLPFC executive systems, the IPS takes on a domaingeneral ability for selection/manipulation of internally-buffered information - which is required in many episodic and semantic tasks. Indeed, previous studies of episodic retrieval have also suggested that the IPS plays an executive role in decision-making during episodic tasks $(4,50)$. Overall, we propose that episodic retrieval is an active construction process where the memory is reconstructed online thus needs both buffering of info from episodic-related areas and also executively-demanding shaping, thereby recruiting both dLPC and vLPC systems.

\section{A unifying account of lateral parietal cortex functions:}

By exploring both the variation in functional response and connectivity profiles across the LPC, the current study found evidence in favour of the neurocomputational principles embraced by the PUCC model $(1,10,35)$. A key notion is that the expressed functions of a cortical region will reflect the combination of the local computation and its pattern of connectivity. This tenant has been formally demonstrated by computational models, whereby the resultant "behaviour" of a processing unit depends not only on its local computations, but also on its long-range connectivity (more recently referred to as 'connectivity-constrained cognition-C3': (51-53)). How this might apply to the LPC is sketched out in Figure 4. In its most simple form (Panel B), the LPC might have a single local computation - an online multi-modal buffer of internal and external sequentially-experienced information. Computational models with recurrent connections (shown as a generic 'Elman' network in Fig 4B) show this general property and the same type of model can be applied to any time-extended series of inputs and outputs $(54,55)$. Accordingly, across a larger region such as the LPC, even if the local buffering computation itself was the same, the emergent observed function will depend on what sources of information and influences arrive at each subregion: i.e., the differential connectivity pattern. Figure 4.B shows a schematic of the varying pattern of connectivity to LPC subregions. We 
can consider the influence of this connectivity profile in two steps. First (Figure 4.C), the long-range connectivity from executively-related DLPFC primarily terminates in the IPS/dorsal LPC region (56) and not the ventral parietal cortex (VPC). This should generate a fundamental, counterpointed difference in the emergent observed functions: in receiving top-down signals from frontal executive processing areas, dLPC (IPS) will operate as a domain-general executive system engaged in the selection/manipulation processes on internally-buffered information $(1,18,20)$. The IPS region itself connects to all subregions of the VPC and thus can become involved in any particular activity irrespective of information type (c.f., the DLPFC and IPS are two key components of the "multidemand' system: $(20,57)$. In contrast to the IPS, most of the VPC does not seem to receive this same level of DLPFC connectivity and thus its underlying buffering will not be so executively penetrated; i.e., its buffering will be more "automatic" (e.g., a distinction envisaged in the differentiation between the central executive and 'slave' subsystems in classical models of short-term working memory: (5860).. More generally the differential connectivity of DLPFC to IPS and not VPC might also help to explain why in many situations dLPC and VLPC show anti-correlated functional activity (18, 22-24); whereby the dLPC is increasingly activated and the VLPC increasingly deactivated based on task difficulty (18). When engaged in a goal-oriented task, on-going automatic information accumulation in VPC subregions would presumably be unhelpful/disruptive unless this input is necessary for task performance. In this case, activation in the irrelevant VPC subregions would be suppressed/deactivated (35). This would be especially needed with increasing demands on task performance, thereby explaining the common observed pattern of anticorrelated activation modulated by task difficulty (18).

Different functions across the VPC itself should emerge given that there are graded differences in connectivity of VPC subregions to distinct neural networks (Fig. 4.D) $(1,35,61,62)$. Specifically, the more anterior VPC through its primary connections to pSTG and pMTG becomes involved in sound, phonological and language processing $(1,63)$ The most posterior VPC subregion is the most heavily influenced by the dorsal connectivity from visual regions, consistent with its involvement in visuospatial processing $(1,2,18,34,35)$, whilst the mid-AG region's involvement in episodic tasks seems entirely consistent with its connectivity through to key nodes of the extended episodic network $(32,33)$.

To conclude, we propose a unified model of LPC function in LPC acts as multi-modal buffer of information. Despite a common core mechanism, graded subdivisions in how this function is expressed can arise based on varying long-range connectivity inputs to dorsal-ventral and anteriorposterior areas.

\section{Methods}


fMRI study

\section{Participants}

Twenty-two participants took part in the fMRI study (average age $=23.81, \mathrm{SD}=4.54 ; \mathrm{N}$ female $=15$ ). All participants were native English speakers with no history of neurological or psychiatric disorders and normal or corrected-to-normal vision.

\section{Task design and procedures}

There were four experimental tasks: episodic, semantic, picture-decision, and control. In each task the participant was presented with word-triads including a target word in the centre of the screen and two words below, one on the left and one on the right. The participants had to select the correct option by button-press. The words were presented on top of a scrambled or unscrambled picture depending on the condition. The trials lasted 4 seconds and were preceded by an instruction indicating the upcoming task. The trials were presented using an event-related design with the most efficient ordering of events determined using Optseq (http://www.freesurfer. net/optseq). Null time was intermixed between trials and varied between 0 and 26 seconds (average $=1.80$ seconds, $\mathrm{SD}=3.13$ ) during which a fixation cross was presented. The experiment was presented in three runs, each lasting 620 seconds, the order of which was counterbalanced across participants. An example trial from each task can be seen in Figure1.

Semantic task: Here the participants were presented with a target word (e.g., daffodil) and two-alternative possible features of the object (e.g., yellow and red). The words were presented on top of a scrambled picture. The participants were instructed to determine which alternative was correct.

Picture-decision task: Here the word triads were presented on top of a colour photograph of an object (e.g., a chair). The target word referred to a property of the picture (e.g., colour) and two alternative choices (e.g., blue and red). The participants were instructed to select the option that best matched the target feature of the object.

Episodic task: Immediately prior to the scan the participants were exposed to a selection of colour photographs of objects (e.g., a bucket) and told that they would be required to remember aspects of the pictures during the experiment. Each photograph was presented for 10 seconds and the participants were asked to describe the picture in as much detail to ensure that the pictures were sufficiently encoded. In each trial of the experiment a target word would be presented (e.g., bucket) and the two alternative possible features of the remembered item (e.g., green or red). The words were presented on top of a scrambled picture. The participants were instructed recall the feature that best described the target item. After a short jittered interval (varying from 0-1.5 seconds) the participants were asked to rate the vividness of their memory of that particular item from 1-4 $(1=$ not vivid, $4=$ very vivid). 
Control task: In the control task the word-triads consisted of a string of Xs (e.g., xxxxxxxxx) on top of a scrambled picture. The picture was shifted slightly to the left or right. The participants had to indicate the direction of the shift. This acted as control for visual and motor activation.

\section{fMRI acquisition parameters}

Images were acquired using a $3 \mathrm{~T}$ Philips Achieva scanner using a dual gradient-echo sequence, which has improved signal relative to conventional techniques, especially in areas associated with signal loss (64). 31 axial slices were collected using a TR $=2.8$ seconds, $\mathrm{TE}=12$ and $35 \mathrm{~ms}$, flip angle $=95^{\circ}, 80 \times 79$ matrix, with resolution $3 \times 3 \mathrm{~mm}$, slice thickness $4 \mathrm{~mm}$. B0 images were also acquired to correct for image distortion.

\section{$\underline{\text { fMRI data analysis }}$}

Preprocessing: The dual-echo images were first B0 corrected and then averaged. Data were analysed using SPM8. Images were motion-corrected and co-registered to the participants T1 structural image, and then spatially normalised into MNI space using DARTEL (65). The functional images were then resampled to a $3 \times 3 \times 3 \mathrm{~mm}$ voxel size and smoothed with an $8 \mathrm{~mm}$ FWHM Gaussian kernel.

General Linear Modelling: The data were filtered using a high-pass filter with a cut-off of 190s and then analysed using a general linear model. At the individual subject level, each condition was modelled with a separate regressor and events were convolved with the canonical hemodynamic response function. Time and dispersion derivatives were added and motion parameters were entered into the model as covariates of no interest. At the individual level, each task condition was modelled separately against rest. A standard voxel height threshold $\mathrm{p}<.001$, cluster corrected using FWE $\mathrm{p}<$ .05 was used. The specific hypothesis regarding the AG and IPS were tested using ROIs taken from a previous large-scale multi-domain meta-analysis (1) Note, the AG ROI corresponded to a $8 \mathrm{~mm}$ sphere centred on the coordinates showing maximum likelihood of activation for both semantic and episodic meta-analyses, and two regions showing domain-general executive processing in the IPS (the pattern of results was the same in both IPS ROIs) (1).

\section{Distortion-corrected diffusion weighted imaging and probabilistic fibre tracking}

Diffusion-weighted images were acquired in 24 healthy volunteers (11 females; mean age 25.9 , range 19-47) without any record of neurological or psychiatric disorders. This dataset has described previously and utilized for various tractography-related explorations (66-71). All participants were right-handed, as assessed by the Edinburgh Handedness Inventory (72). Participants gave written informed consent to the study protocol, which had been approved by the local ethics committee. 
A 3 T Philips Achieva scanner (Philips Medical System, Best Netherlands) was used for acquiring imaging data with an eight-channel SENSE head coil. Diffusion weighted imaging (DWI) was performed using a pulsed gradient spin echo-planar sequence, with $\mathrm{TE}=59 \mathrm{~ms}, \mathrm{TR} \approx 11,884 \mathrm{~ms}$, $\mathrm{G}=62 \mathrm{mTm}^{-1}$, half scan factor $=0.679,112 \times 112$ image matrix reconstructed to $128 \times 128$ using zero padding, reconstructed resolution $1.875 \times 1.875 \mathrm{~mm}$, slice thickness $2.1 \mathrm{~mm}, 60$ contiguous slices, 61 non-collinear diffusion sensitization directions at $\mathrm{b}=1200 \mathrm{smm}^{-2}(\Delta=29.8 \mathrm{~ms}, \delta=13.1 \mathrm{~ms}), 1$ at $\mathrm{b}=0$, SENSE acceleration factor $=2.5$. Acquisitions were cardiac gated using a peripheral pulse unit positioned over the participants' index finger or an electrocardiograph. For each gradient direction, two separate volumes were obtained with opposite polarity $k$-space traversal with phase encoding in the left-right/right-left direction to be used in the signal distortion correction procedure (73). A colocalized T2 weighted turbo spin echo scan was acquired with in-plane resolution of $0.94 \times 0.94 \mathrm{~mm}$ and slice thickness $2.1 \mathrm{~mm}$, as a structural reference scan to provide a qualitative indication of distortion correction accuracy. A high-resolution T1-weighted 3D turbo field echo inversion recovery image $\left(\mathrm{TR} \approx 2000 \mathrm{~ms}, \mathrm{TE}=3.9 \mathrm{~ms}, \mathrm{TI}=1150 \mathrm{~ms}\right.$, flip angle $8^{\circ}, 256 \times 205$ matrix reconstructed to $256 \times 256$, reconstructed resolution $0.938 \times 0.938 \mathrm{~mm}$, slice thickness $0.9 \mathrm{~mm}, 160$ slices, SENSE factor $=2.5$ ), was obtained for the purpose of high-precision anatomical localization of seed regions for tracking.

Four independently defined regions of interest (ROIs) were identified for tractography: there was one dLPC (IPS) ROI located in an area of domain-general executive processing based on metaanalysis data (1). The vLPC was divided into three ROIs based on the ICA results from a previous independent fMRI study which divided the AG into three differential regions along an anteriorposterior axis: anterior-vLPC (PGa), mid-vLPC (PGp), and posterior-vLPC (PGp) (35) Note that the mid-LPC corresponds most closely to the DMN area, although the entire AG is often implicated. Previous research has shown that these ROIs show functionally distinct responses to different fMRI task modalities, with the anterior region responding positively to sentences, and showing strong functional connectivity with the fronto-temporal language network, the mid-region showing no task preference (equal deactivation) and functionally connecting to the $\mathrm{DMN}$, and the posterior region responding positively to picture stimuli and showing functional connectivity with visual cortex (35). ROIs were defined as an $8 \mathrm{~mm}$ radius sphere in the left hemisphere and transformed into each individual's native diffusion space using the diffeomorphic anatomical registration through an exponentiated lie algebra (DARTEL) toolbox (65) based on each participant's T1-weigthed images.

Unconstrained probabilistic tractography was performed using the PICo software package (74), sampling the orientation of probability density functions (PDFs) generated constrained spherical deconvolution (CSD) (75) and model-based residual bootstrapping (76, 77). 20,000 Monte Carlo streamlines were initiated from each voxel within an ROI. Step size was set to $0.5 \mathrm{~mm}$. Stopping criteria for the streamlines were set so that tracking terminated if pathway curvature over a voxel was greater than $180^{\circ}$, or the streamline reached a physical path limit of $500 \mathrm{~mm}$. 
The tracking results for each participants were spatially normalized into MNI space using the DARTEL toolbox. The brain regions associated with each fibre pathway were determined using brain masks from the AAL atlas or the Juelich histological atlas, which has a finer demarcation for the parietal cortex. The superior, middle, and inferior temporal lobe were further subdivided into anterior, middle, and posterior regions based on those used previously (69) since these regions have been shown to have large variations in connectivity profiles $(69,71)$. For each ROI, the atlas masks were overlaid over each participant's tracking data and a maximum connectivity value (ranging from 0 to 20,000 ) between the ROI and each region of the brain was estimated. Thereby, we obtained a single probability estimate of a pathway between each pair of regions. These values were placed into an individual-specific matrix. Then, the connectivity matrices were subjected to a double threshold to ensure that only connections with high probability in the majority of participants were considered. For the first-level individual threshold, following the approach described by Cloutman et al (67), the $\lambda$ value of the Poisson distribution identified was used to determine a threshold value at $p=0.025$. For the second-level group threshold, we used both a stringent (over $70 \%$ of participants, i.e., at least $17 / 24$ participants) and a more relaxed (over $50 \%$ of participants, i.e., at least $12 / 24$ participants) criteria for consistency.

\section{Acknowledgements:}

This research was supported by an MRC Programme grant to MALR (MR/R023883/1).

\section{References}

1. Humphreys GF \& Lambon Ralph MA (2015) Fusion and fission of cognitive functions in the human parietal cortex. Cerebral Cortex 25:3547-3560.

2. Corbetta M \& Shulman GL (2002) Control of goal-directed and stimulus-driven attention in the brain. Nat Rev Neurosci 3(3):201-215.

3. Cabeza R, Ciaramelli E, \& Moscovitch M (2012) Cognitive contributions of the ventral parietal cortex: an integrative theoretical account. Trends in Cognitive Sciences 16(6):338352.

4. Sestieri C, Shulman GL, \& Corbetta M (2017) The contribution of the human posterior parietal cortex to episodic memory. Nature Reviews Neuroscience 18:183-192.

5. Binder JR, Desai RH, Graves WW, \& Conant L (2009) Where Is the Semantic System? A Critical Review and Meta-Analysis of 120 Functional Neuroimaging Studies. Cerebral Cortex 19(12):2767-2796.

6. Wagner AD, Shannon BJ, Kahn I, \& Buckner RL (2005) Parietal lobe contributions to episodic memory retrieval. Trends in Cognitive Sciences 9(9):445-453.

7. Buckner RL, Andrews-Hanna JR, \& Schacter DL (2008) The brain's default network Anatomy, function, and relevance to disease. Ann Ny Acad Sci 1124:1-38.

8. Humphreys GF, Hoffman P, Visser M, Binney RJ, \& Ralph MAL (2015) Establishing taskand modality-dependent dissociations between the semantic and default mode networks. Proceedings of the National Academy of Sciences of the United States of America 112(25):7857-7862. 
9. Raichle ME, et al. (2001) A default mode of brain function. Proceedings of the National Academy of Sciences 98(2):676-682.

10. Humphreys GF, Lambon Ralph MA, \& Simons JS (2020) A unifying account of angular gyrus contributions to episodic and semantic cognition. PsyArXiv 26.

11. Caspers S, et al. (2008) The human inferior parietal lobule in stereotaxic space. Brain Structure \& Function 212(6):481-495.

12. Caspers S, et al. (2011) Probabilistic fibre tract analysis of cytoarchitectonically defined human inferior parietal lobule areas reveals similarities to macaques. Neuroimage 58(2):362380 .

13. Uddin LQ, et al. (2010) Dissociable Connectivity within Human Angular Gyrus and Intraparietal Sulcus: Evidence from Functional and Structural Connectivity. Cerebral Cortex 20(11):2636-2646.

14. Cloutman LL, Binney RJ, Morris DM, Parker GJM, \& Lambon Ralph MA (2013) Using in vivo probabilistic tractography to reveal two segregated dorsal 'language-cognitive' pathways in the human brain. Brain Lang 127:230-240.

15. Geschwind N (1972) Language and Brain. Sci Am 226(4):76-\&.

16. Vilberg KL \& Rugg MD (2008) Memory retrieval and the parietal cortex: A review of evidence from a dual-process perspective. Neuropsychologia 46(7):1787-1799.

17. Renoult L, Irish M, Moscovitch M, \& Rugg MD (2019) From Knowing to Remembering: The Semantic-Episodic Distinction. Trends Cogn Sci 23(12):1041-1057.

18. Humphreys GF \& Lambon Ralph MA (2017) Mapping Domain-Selective and Counterpointed Domain-General Higher Cognitive Functions in the Lateral Parietal Cortex: Evidence from fMRI Comparisons of Difficulty-Varying Semantic Versus Visuo-Spatial Tasks, and Functional Connectivity Analyses. Cerebral Cortex 27(8):4199-4212.

19. Walsh V (2003) A theory of magnitude: common cortical metrics of time, space and quantity. Trends in Cognitive Sciences 7:483-488.

20. Fedorenko E, Duncan J, \& Kanwisher N (2013) Broad domain generality in focal regions of frontal and parietal cortex. Proc Natl Acad Sci U S A 110(41):16616-16621.

21. Andrews-Hanna JR (2012) The Brain's Default Network and Its Adaptive Role in Internal Mentation. Neuroscientist 18(3):251-270.

22. Keller CJ, et al. (2013) Neurophysiological Investigation of Spontaneous Correlated and Anticorrelated Fluctuations of the BOLD Signal. Journal of Neuroscience 33(15):6333-6342.

23. Chai XQJ, Castanon AN, Ongur D, \& Whitfield-Gabrieli S (2012) Anticorrelations in resting state networks without global signal regression. Neuroimage 59(2):1420-1428.

24. Fox MD, Zhang DY, Snyder AZ, \& Raichle ME (2009) The Global Signal and Observed Anticorrelated Resting State Brain Networks. Journal of Neurophysiology 101(6):3270-3283.

25. Pexman PM, Hargreaves IS, Edwards JD, Henry LC, \& Goodyear BG (2007) Neural correlates of concreteness in semantic categorization. Journal of Cognitive Neuroscience 19(8):1407-1419.

26. Graves WW, Boukrina O, Mattheiss SR, Alexander EJ, \& Baillet S (2017) Reversing the Standard Neural Signature of the Word-Nonword Distinction. J Cognitive Neurosci 29(1):7994.

27. Dehaene S, Piazza M, Pinel P, \& Cohen L (2003) Three parietal circuits for number processing. Cognitive Neuropsychology 20(3-6):487-506.

28. Rice GE, Lambon Ralph MA, \& Hoffman P (2015) The roles of left versus right anterior temporal lobes in conceptual knowledge: an ALE meta-analysis of 97 functional neuroimaging studies. Cerebral Cortex 25(11):4374-4391.

29. Rice GE, Hoffman P, \& Lambon Ralph MA (2015) Graded specialization within and between the anterior temporal lobes. Annals of the New York Academy of Sciences 1359(1):84-97.

30. Lambon Ralph MA, Jefferies E, Patterson K, \& Rogers TT (2017) The neural and computational bases of semantic cognition. Nat Rev Neurosci 18(1):42-55.

31. Vigneau M, et al. (2006) Meta-analyzing left hemisphere language areas: phonology, semantics, and sentence processing. Neuroimage 30(4):1414-1432.

32. Rugg MD \& Vilberg KL (2013) Brain networks underlying episodic memory retrieval. Current Opinion in Neurobiology 23(2):255-260. 
33. Sestieri C, Corbetta M, Romani GL, \& Shulman GL (2011) Episodic Memory Retrieval, Parietal Cortex, and the Default Mode Network: Functional and Topographic Analyses. Journal of Neuroscience 31(12):4407-4420.

34. Zacks JM (2008) Neuroimaging studies of mental rotation: A meta-analysis and review. Journal of Cognitive Neuroscience 20(1):1-19.

35. Humphreys GF, Jackson RL, \& Lambon Ralph MA (2020) Overarching Principles and Dimensions of the Functional Organization in the Inferior Parietal Cortex. Cerebral Cortex 30(11):5639-5653.

36. Branzi FM, Humphreys GF, Hoffman P, \& Lambon Ralph MA (2020) Revealing the neural networks that extract conceptual gestalts from continuously evolving or changing semantic contexts. Neuroimage 220:116802.

37. Visser M, Jefferies E, \& Lambon Ralph MA (2010) Semantic processing in the anterior temporal lobes: A meta-analysis of the functional neuroimaging literature. Journal of Cognitive Neuroscience 22(6):1083-1094.

38. Geranmayeh F, et al. (2012) The contribution of the inferior parietal cortex to spoken language production. Brain and Language 121(1):47-57.

39. Binder JR, et al. (1999) Conceptual processing during the conscious resting state: A functional MRI study. J Cognitive Neurosci 11(1):80-93.

40. Mason MF, et al. (2007) Wandering minds: the default network and stimulus-independent thought. Science 315(5810):393-395.

41. Hahn B, Ross TJ, \& Stein EA (2007) Cingulate activation increases dynamically with response speed under stimulus unpredictability. Cerebral Cortex 17(7):1664-1671.

42. Berryhill ME, Phuong L, Picasso L, Cabeza R, \& Olson IR (2007) Parietal lobe and episodic memory: Bilateral damage causes impaired free recall of autobiographical memory. $J$ Neurosci 27:14415-14423.

43. Simons JS, et al. (2008) Is the parietal lobe necessary for recollection in humans? Neuropsychologia 46:1185-1191.

44. Bonnici HM, Cheke LG, Green DAE, FitzGerald THMB, \& Simons JS (2018) Specifying a causal role for angular gyrus in autobiographical memory. J Neurosci 38:10438-10443.

45. Davidson PSR, et al. (2008) Does lateral parietal cortex support episodic memory? Evidence from focal lesion patients. Neuropsychologia 46:1743-1755.

46. Yazar Y, Bergstrom ZM, \& Simons JS (2014) Continuous theta burst stimulation of angular gyrus reduces subjective recollection. Plos One 9(10):e110414.

47. Moscovitch M, Cabeza R, Winocur G, \& Nadel L (2016) Episodic memory and beyond: The hippocampus and neocortex in transformation. Annual Review of Psychology 67:105-134.

48. Shimamura AP (2011) Episodic retrieval and the cortical binding of relational activity. Cogn Affect Behav Neurosci 11(3):277-291.

49. St. Jacques PL (2019) A New Perspective on Visual Perspective in Memory. Current Directions in Psychological Science:0963721419850158.

50. Gonzalez AJ, et al. (2015) Parietal dynamics during recognition memory. P Natl Acad Sci USA 112(35):11066-11071.

51. Lambon Ralph MA, McClelland JL, Patterson K, Galton CJ, \& Hodges JR (2001) No right to speak? The relationship between object naming and semantic impairment: neuropsychological evidence and a computational model. J Cognitive Neurosci 13:341-356.

52. Plaut DC (2002) Graded modality-specific specialization in semantics: A computational account of optic aphasia. Cognitive Neuropsychology 19:603-639.

53. Chen L, Lambon Ralph MA, \& Rogers TT (2017) A unified model of human semantic knowledge and its disorders. Nat Hum Behav 1:1-10.

54. Botvinick MM \& Plaut DC (2006) Short-term memory for serial order: A recurrent neural network model. Psychol Rev 113(2):201-233.

55. Elman JL (1990) Finding Structure in Time. Cognitive Science 14:179-211.

56. Crowe DA, et al. (2013) Prefrontal neurons transmit signals to parietal neurons that reflect executive control of cognition. Nat Neurosci 16:1484-1491.

57. Assem M, Glasser MF, Van Essen DC, \& Duncan J (2020) A domain-general cognitive core defined in multimodally parcellated human cortex. Cerebral Cortex 30(8):4361-4380. 
58. Baddeley A (2000) The episodic buffer: A new component of working memory? Trends Cogn Sci 4:417-423.

59. Baddeley A (2003) Working memory: looking back and looking forward. Nature Reviews Neuroscience 4(10):829-839.

60. Broadbent DE (1982) Task combination and selective intake of information. Acta Psychologica 50(3):253-290.

61. Nelson SM, McDermott KB, \& Petersen SE (2012) In favor of a 'fractionation' view of ventral parietal cortex: comment on Cabeza et al. Trends Cogn Sci 16(8):399-400; author reply 400-391.

62. Daselaar SM, Huijbers W, Eklund K, Moscovitch M, \& Cabeza R (2013) Resting- state functionl connectivity of ventral parietal regions associated with attention reorienting and episodic recollection. Frontiers in Human Neuroscience 7.

63. Griffiths TD \& Warren JD (2002) The planum temporale as a computational hub. Trends in Neurosciences 25:348-353.

64. Halai AD, Welbourne SR, Embleton K, \& Parkes LM (2014) A comparison of dual gradientecho and spin-echo fMRI of the inferior temporal lobe. Human Brain Mapping 35(8):41184128.

65. Ashburner J (2007) A fast diffeomorphic image registration algorithm. Neuroimage 38(1):95113.

66. Bajada CJ, et al. (2016) The tract terminations in the temporal lobe: Their location and associated functions. Cortex.

67. Cloutman LL, Binney RJ, Drakesmith M, Parker GJ, \& Lambon Ralph MA (2012) The variation of function across the human insula mirrors its patterns of structural connectivity: evidence from in vivo probabilistic tractography. Neuroimage 59(4):3514-3521.

68. Binney RJ, Parker GJ, \& Lambon Ralph MA (2012) Convergent connectivity and graded specialization in the rostral human temporal lobe as revealed by diffusion-weighted imaging probabilistic tractography. J Cogn Neurosci 24(10):1998-2014.

69. Jung J, Cloutman LL, Binney RJ, \& Lambon Ralph MA (2017) The structural connectivity of higher order association cortices reflects human functional brain networks. Cortex 97:221239.

70. Jung J, Visser M, Binney RJ, \& Lambon Ralph MA (2018) Establishing the cognitive signature of human brain networks derived from structural and functional connectivity. Brain Struct Funct 223(9):4023-4038.

71. Bajada CJ, et al. (2017) A graded tractographic parcellation of the temporal lobe. Neuroimage 155:503-512.

72. Oldfield RC (1971) The assessment and analysis of handedness: the Edinburgh inventory. Neuropsychologia 9(1):97-113.

73. Embleton KV, Haroon HA, Morris DM, Ralph MA, \& Parker GJ (2010) Distortion correction for diffusion-weighted MRI tractography and fMRI in the temporal lobes. Hum Brain Mapp 31(10):1570-1587.

74. Parker GJ, Haroon HA, \& Wheeler-Kingshott CA (2003) A framework for a streamline-based probabilistic index of connectivity (PICo) using a structural interpretation of MRI diffusion measurements. J Magn Reson Imaging 18(2):242-254.

75. Tournier JD, et al. (2008) Resolving crossing fibres using constrained spherical deconvolution: validation using diffusion-weighted imaging phantom data. Neuroimage 42(2):617-625.

76. Haroon HA, Morris DM, Embleton KV, Alexander DC, \& Parker GJ (2009) Using the model-based residual bootstrap to quantify uncertainty in fiber orientations from Q-ball analysis. IEEE Trans Med Imaging 28(4):535-550.

77. Jeurissen B, Leemans A, Jones DK, Tournier JD, \& Sijbers J (2011) Probabilistic fiber tracking using the residual bootstrap with constrained spherical deconvolution. Hum Brain Mapp 32(3):461-479. 
bioRxiv preprint doi: https://doi.org/10.1101/2020.12.08.416123; this version posted December 8, 2020. The copyright holder for this preprint (which was not certified by peer review) is the author/funder, who has granted bioRxiv a license to display the preprint in perpetuity. It is made available under aCC-BY-NC-ND 4.0 International license. 
Episodic trial

Semantic trial

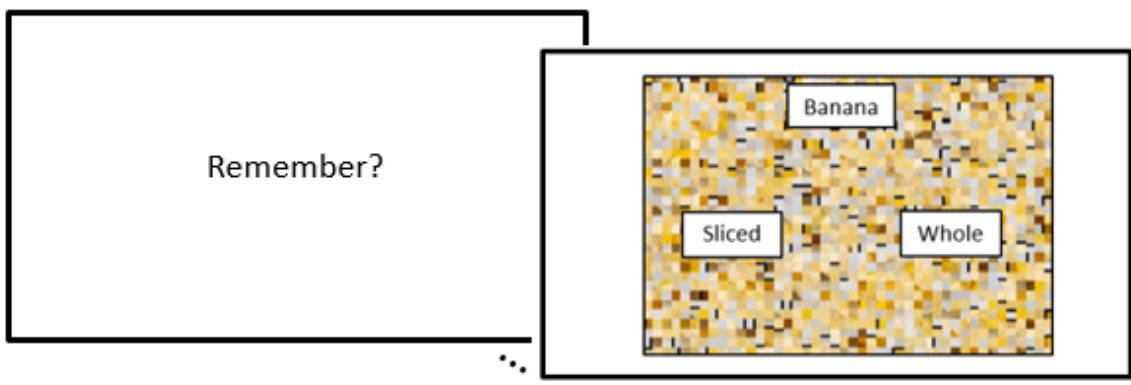

Semantic trial

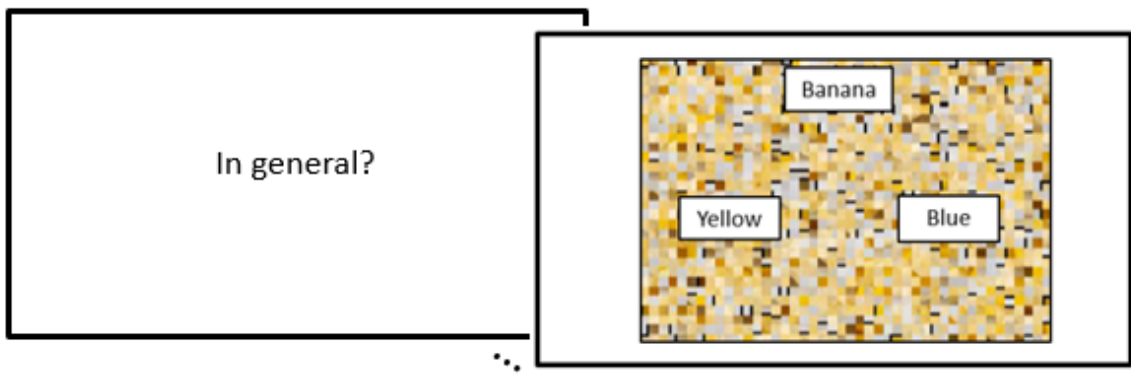

Picture-decision trial

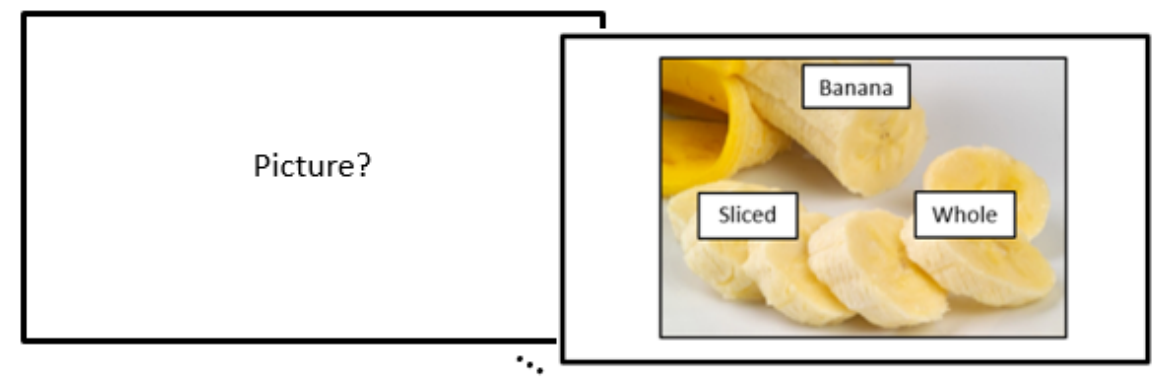

\section{Control trial}

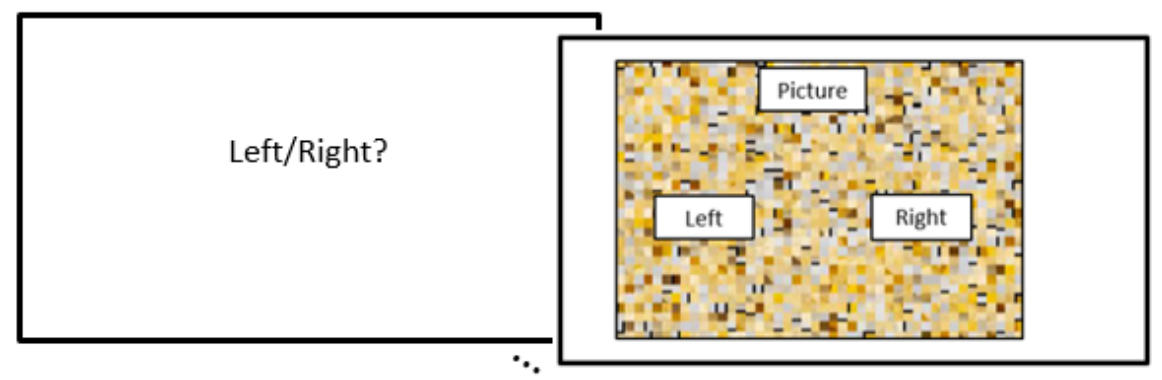

Figure 1: One example trial from each experimental condition 


\section{a) Episodic > Control (red), Semantic > Control (blue)}

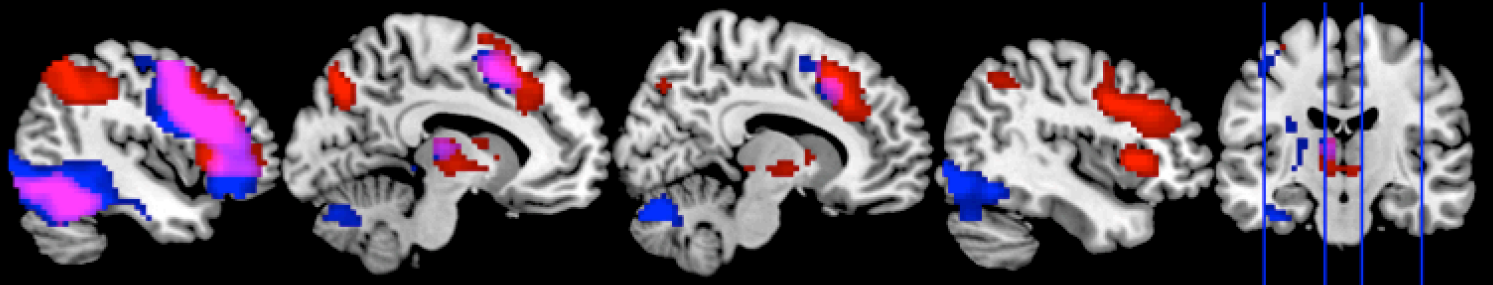

b) Episodic > Semantic (red), Semantic > Episodic (blue)

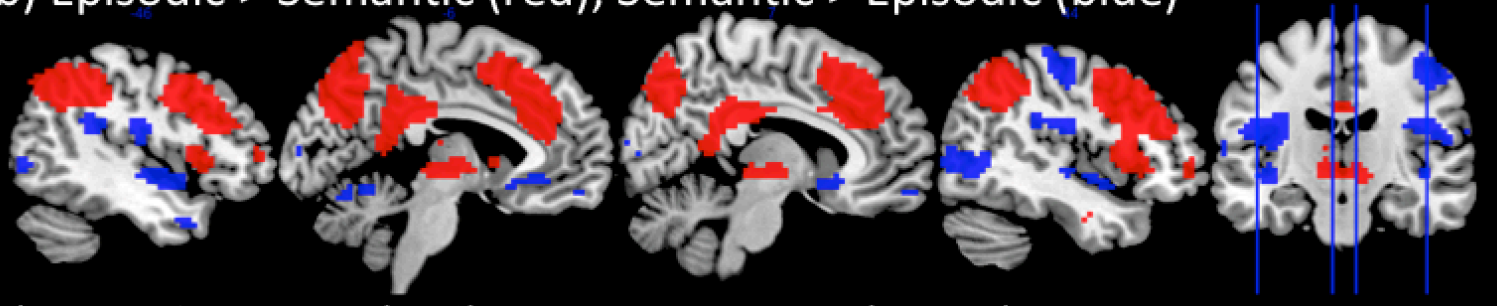

c) Episodic > Rest (red), Rest $>$ Semantic (green)

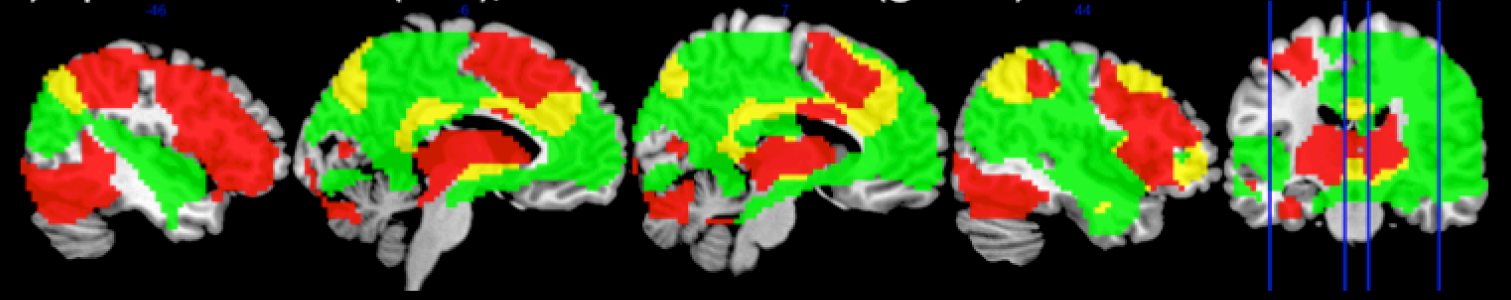

d) Positive correlations with vividness in episodic retrieval task

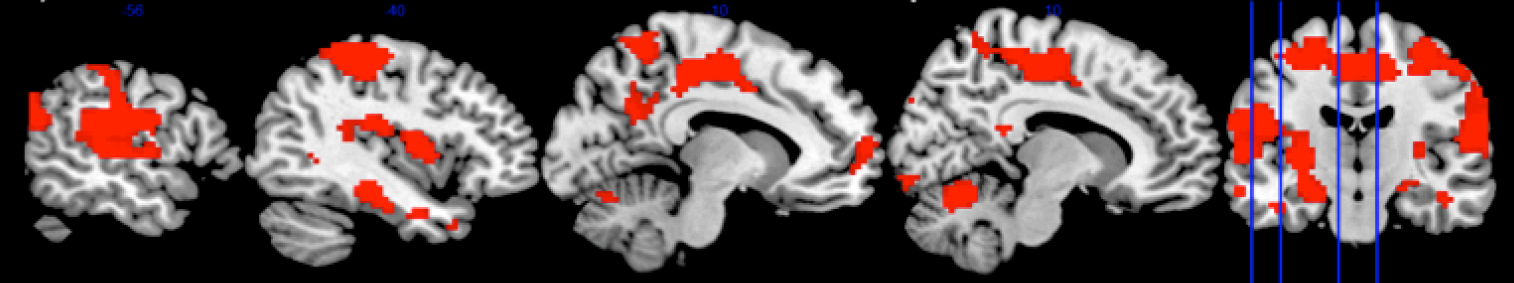

e) Positive (red) and Negative (blue) correlations with RT

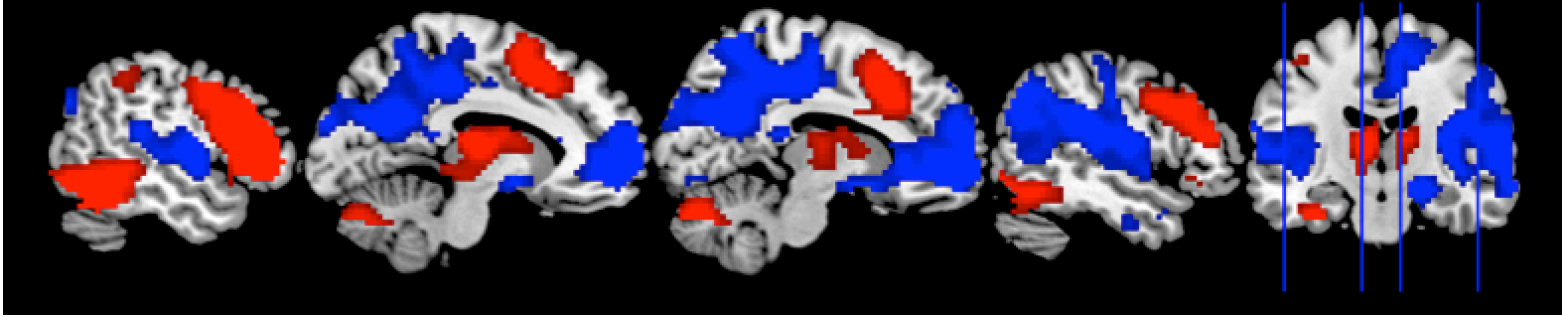

Figure 2. A: Whole-brain responses to the contrast of Episodic $>$ Control (red) and Semantic $>$

Control (blue). B: Whole-brain responses to the contrast of Episodic $>$ Semantic (red) and Semantic $>$ Episodic (blue). $C$ : Whole-brain responses showing positive activation relative to rest for the episodic task (Episodic $>$ Rest (red)), and deactivation relative to rest for the semantic task (Rest $>$ Semantic (green)). This shows positive LTC activation for the episodic task but deactivation for the semantic task. $D$ : The areas that positively correlated with vividness ratings during the episodic retrieval task. $E$ : The network showing a positive correlation (red) and a negative correlation (blue) with task difficulty. Analyses were performed using a standard voxel height threshold $\mathrm{p}<.001$, cluster corrected using FWE $\mathrm{p}<.05$. 


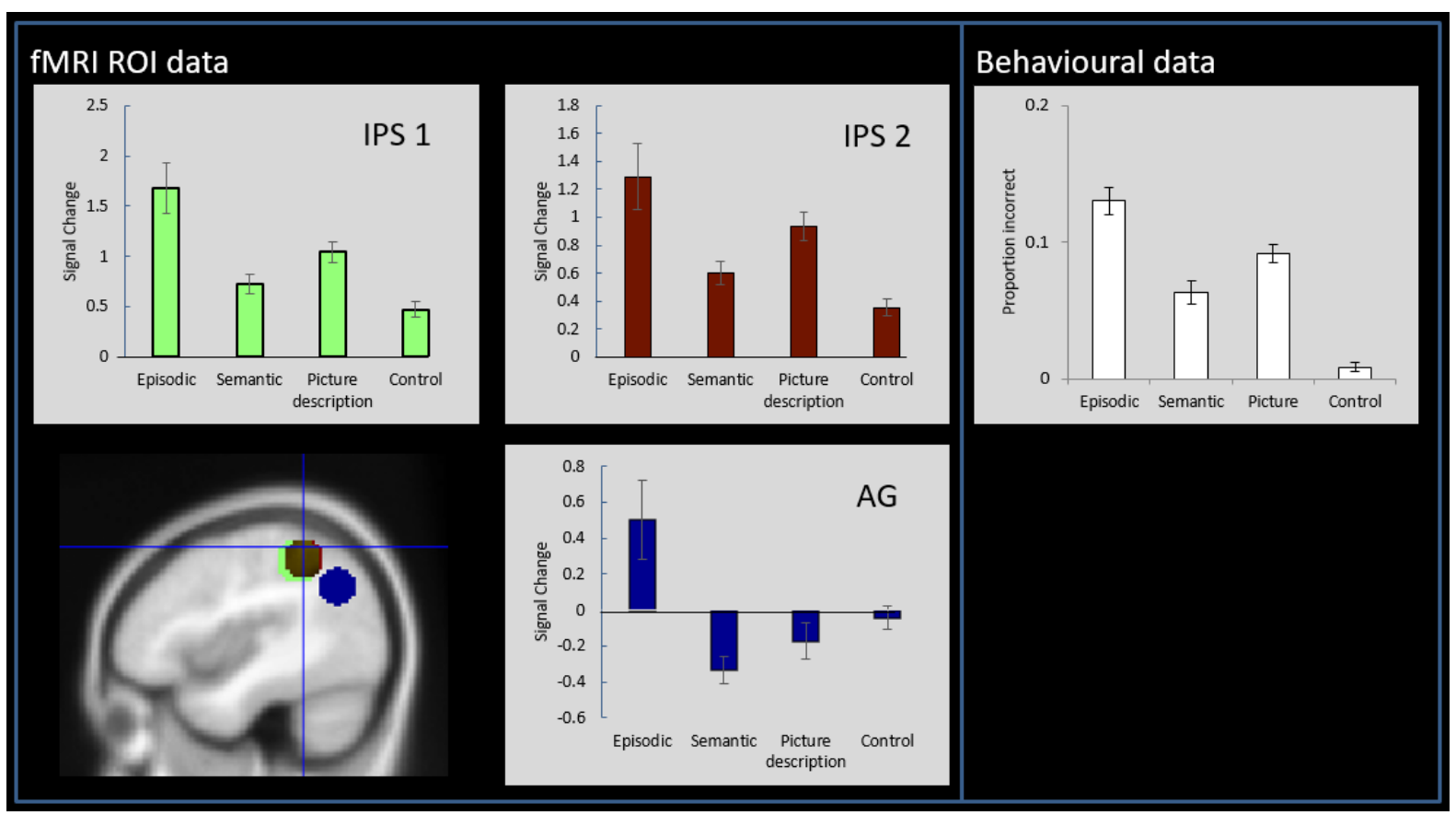

Figure 3. Left panel: ROI analyses showing mean activation relative to rest for each condition within the IPS and AG. The ROIs were defined based on the results from a large-scale multidomain metaanalysis. Right panel: The behavioural data showing the average proportion of incorrect responses during the fMRI task. Note. The behavioural data closely mirrors the fMRI activity in the IPS. 


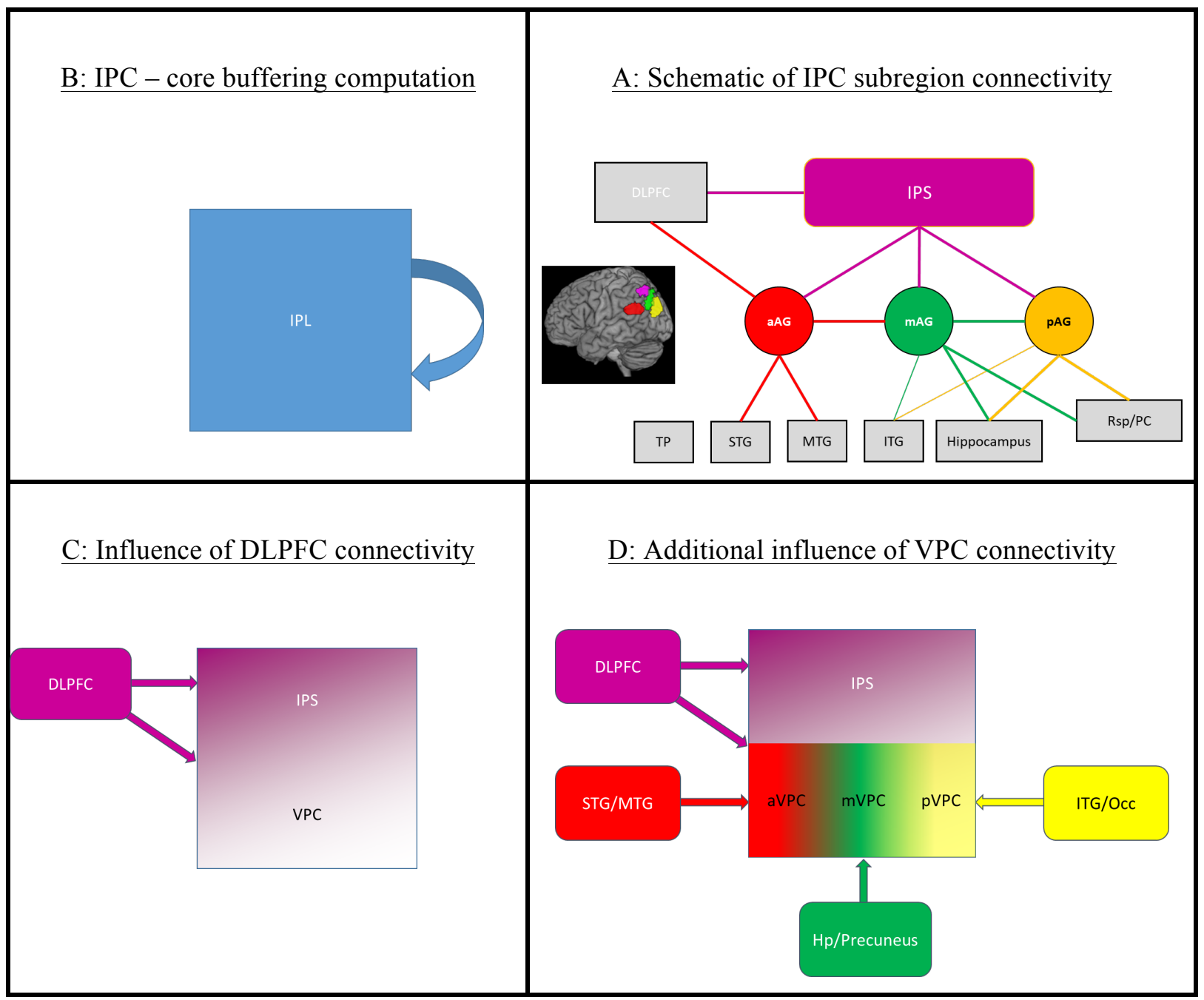

Figure 4. A schematic illustration of the varying long-range connectivity inputs and their consequential influence on the emergent IPC functions. 
Table1. Group-level connectivity matrix. Bold font indicates that the connection probability was over $70 \%$ for the group analysis. The individual threshold was set at $2.5 \%$.

\begin{tabular}{|c|c|c|c|c|c|}
\hline & & Dorsal & & Ventral & \\
\hline & & IPS & Anterior & Middle & Posterior \\
\hline $\begin{array}{l}\text { Temporal } \\
\text { lobe }\end{array}$ & mMTG & & 92 & & \\
\hline & $\mathrm{pSTG}$ & & 96 & & \\
\hline & pMTG & & 96 & & \\
\hline & pITG & & & 58 & 54 \\
\hline & Hippocampus & & & 83 & 92 \\
\hline Frontal lobe & DLPFC & 71 & 71 & & \\
\hline & BA44 & & 50 & & \\
\hline Parietal lobe & S1 & 100 & 75 & 88 & 58 \\
\hline & $5 \mathrm{Ci}$ & & & & \\
\hline & $5 \mathrm{M}$ & & & & \\
\hline & $5 \mathrm{~L}$ & & & 63 & \\
\hline & $7 \mathrm{PC}$ & 100 & 63 & 92 & 67 \\
\hline & $7 \mathrm{~A}$ & 96 & & 96 & 79 \\
\hline & $7 \mathrm{P}$ & & & 83 & 71 \\
\hline & $7 \mathrm{M}$ & & & 54 & 75 \\
\hline & IPS1 & 100 & 75 & 92 & \\
\hline & IPS2 & 100 & 67 & & \\
\hline & IPS3 & 100 & & 96 & 63 \\
\hline & PFo & 63 & & & \\
\hline & $\mathrm{PFt}$ & 83 & & & \\
\hline & $\mathrm{PF}$ & 96 & 88 & & \\
\hline & PFm & 75 & 88 & & \\
\hline & $\mathrm{PFcm}$ & 96 & 88 & & \\
\hline & $\mathrm{PGa}$ & 67 & 92 & 88 & \\
\hline & PGp & & 63 & 100 & 83 \\
\hline & Precuneus & & & 88 & 100 \\
\hline $\begin{array}{l}\text { Occipital } \\
\text { lobe }\end{array}$ & Superior OCC & & & 92 & 100 \\
\hline & Middle OCC & 79 & 67 & 100 & 100 \\
\hline & Inferior OCC & & & 50 & 83 \\
\hline
\end{tabular}

